\title{
The 45 and Up Study: a tool for local population health and health service planning to improve integration of healthcare
}

\author{
Elizabeth J Comino ${ }^{a, c}$, Elizabeth Harris ${ }^{a, b}$, Jude Page ${ }^{b}$, Julie McDonalda \\ and Mark F Harrisa
}

a Centre for Primary Health Care and Equity, University of New South Wales, Sydney, Australia

b Health Equity Research \& Development Unit, Sydney Local Health District, NSW, Australia

cCorresponding author: e.comino@unsw.edu.au

\section{Article history}

Publication date: July 2016

Citation: Comino EJ, Harris E, Page J, McDonald J, Harris MF. The 45 and Up Study: a tool for local population health and health service planning to improve integration of healthcare. Public Health Res Pract. 2016;26(3):e2631629. doi: http:// dx.doi.org/10.17061/phrp2631629

\section{Key points}

- The 45 and Up Study is a powerful tool with the capacity to link to a wide number of administrative data collections, enabling the creation of comprehensive data collections across federal and state jurisdictions

- Data from the 45 and Up Study can provide information on the local community and its use of health services, particularly relating to the transfer of care between primary and secondary services, and changes in use over time

- The data collection can address questions of interest to planners working in primary and secondary care, such as integration and coordination of services

\section{Abstract}

Data on patient access to, and use of, primary and secondary care services are a potential tool for population health and health service planning, and for researchers. The Sax Institute's 45 and Up Study was established to support research about healthy ageing. This paper considers how data from the 45 and Up Study could be useful to Local Health Districts and the Primary Health Networks in New South Wales to support their work, particularly in evaluating integration of primary and secondary health services. Preliminary exploration of these data identified more than 31000 participants in the 45 and Up Study living in central and eastern Sydney who were included in some demonstration projects. The value of these data to the region included access to a population-based sample of residents, and capacity to link to health data held within different jurisdictions and local data sources. The resultant data collection can address questions of interest to planners working in primary and secondary care, such as integration and coordination of services, including transition of care. These are key performance goals for both sectors.

\section{Introduction}

The Sax Institute's 45 and Up Study is one of the largest population cohort studies established to date. It comprises more than 267000 participants aged 45 years or over at recruitment from across New South Wales (NSW), Australia. ${ }^{1}$ The 45 and Up Study was established with the capacity to investigate healthy ageing. When they joined the Study, cohort participants gave their approval for the ongoing linkage of their data to available data collections for research purposes until they withdraw from the Study or die. ${ }^{1}$ This large cohort is a potential tool for population health and health services planning, and research into healthy ageing in NSW. Improved linkage capacity, including the establishment of the Centre for Health Record Linkage (CHeReL) to facilitate linkage of available electronic administrative 
data collections, extends the usefulness of the 45 and Up Study for health service research. A limitation of the linked data collections is the focus on the use of services, such as hospitals or medical care, and a lack of clinical information.

Under Australia's universal healthcare system, claims for medical and pharmaceutical care are subsidised by the Australian Government (Department of Human Services Medicare Benefits Schedule [MBS] and Pharmaceutical Benefits Scheme [PBS] $)^{2}$, and public hospital and community health services are provided through state and territory health departments. In NSW, public health services are organised into a number of Local Health Districts (LHDs), which are responsible for providing hospital and community healthcare in defined regions. ${ }^{3}$ Primary Health Networks (PHNs) have been established to coordinate care for patients within community settings. ${ }^{4}$ The LHDs and PHNs have a common interest in improving the health of the population that they serve.

This paper considers how data from the 45 and Up Study could be used to support the work of these organisations in population healthcare planning. The Centre for Primary Health Care and Equity is working with Sydney and South Eastern Sydney LHDs, which share a common catchment with the Central and Eastern Sydney $\mathrm{PHN}$, to explore uses of the 45 and Up Study for local planning purposes. This paper draws on this work.

\section{Imperatives for access to a community-dwelling population}

In recent years, health service developments have aimed to improve the quality of healthcare; reduce costs through better integration and coordination of health services, and information sharing; and provide seamless transitions, particularly between primary and secondary care (especially hospitals). ${ }^{5,6}$ By also strengthening prevention and wellness services, these changes may keep people healthy and reduce their need for acute care services. ${ }^{7}$ Primary care is central to the realisation of these goals. $\mathrm{PHN}$ have coordination of care as a key performance indicator. ${ }^{4}$

It will be challenging for PHNs and LHDs to measure progress on achieving these aims. The lack of shared comprehensive data may limit evaluation to available data within services and about patients who use the services, rather than evaluation of the broader population impact, and change at the interface between primary and secondary care.

\section{How would the 45 and Up Study help?}

In 2015, preliminary discussions were held with Sydney and South Eastern Sydney LHDs to identify interest in, and potential uses of, the 45 and Up Study data at a local level. The LHDs expressed interest in exploring the factors associated with healthy ageing and changing healthcare needs, and better understanding the integration of health services.

Two exploratory linkage studies were undertaken to demonstrate the usefulness of the 45 and Up Study data for this purpose. These data were linked to State (NSW Admitted Patient Data Collection) and MBS data for the 31115 participants who were residing in central or eastern Sydney. The first study was an exploration of enhanced primary care (MBS claims for general practice [GP] management plans, review of GP management plans, continuity of care, and nurse/allied health services) and hospitalisation. Data were extracted for 26429 participants (among total exclusions [ $n=4686$ ] were 4175 participants without a provider number used to calculate continuity of care). Although these enhanced primary care services were provided more frequently to older participants with greater health needs, review of GP management plans was less frequent than what was intended by the relevant health policy. Receipt of these services was associated with reduced likelihood of hospitalisation within the following year. These preliminary findings demonstrated positive benefits of proactive primary care and identified opportunities to improve its implementation among 'at-risk' patients.

A second preliminary study explored time to GP follow-up after hospital discharge. Participants residing in central or eastern Sydney and admitted to hospital in the year following recruitment were eligible $(n=7235)$. Data were linked to the NSW Admitted Patient Data Collection and MBS claims for GP care. Mean time to GP follow-up was 35 days (standard deviation 46 days); 35\% were followed up within 2 weeks (timely). Age (older), poor health status and low socio-economic status (measured from the baseline 45 and Up Study questionnaire) were associated with timely follow-up, suggesting GP return according to healthcare need. These data suggest issues with current discharge policies for follow-up within 7 days. The findings have informed discussions about discharge planning.

\section{Value of a longitudinal study for health services}

These data have a number of benefits for LHDs and $\mathrm{PHNs}$. Within the central and eastern Sydney region, the 45 and Up Study includes 31173 participants (18 595 in the South Eastern Sydney LHD; 12578 in the Sydney LHD). The full 45 and Up Study data come from a variety of settings, and reflect variations across NSW in participant characteristics and healthcare provision, including access to GPs and major health facilities. The central and eastern Sydney data may represent a sizeable, more homogeneous population, with similar characteristics and access to health services. Access to 
longitudinal linked data based on the 45 and Up Study provides access to a population instead of a clinic-based cohort that would be beyond the resources and remit of the organisations to establish.

The 45 and Up Study was designed for record linkage. When establishing the Study, agreement was reached with the Australian Government for access to claims data, including the MBS and PBS, for ongoing linkage. By including the linked data held in NSW (e.g. the Admitted Patient Data Collection), combined national and state data were available. For the first time, these data provided the LHDs and PHNs with information about participants' use of primary and secondary health services. The data provide significant opportunities to examine questions of specific interest.

Locally, health services collect a range of data about their patients. The potential to include these collections in the linkages will enable exploration of specific questions of local relevance, particularly questions about changing health needs and service use.

Healthcare providers are concerned about protecting the privacy of their patients' health records. The use of a third party (the Sax Institute or CHeReL) to organise the linkage enables the provision of de-identified data from different sources for linkage studies. These facilities are further improved with the use of a secure remote data laboratory, the Sax Institute's Secure Unified Research Environment (SURE), with access to unit record data restricted to data analysts.

\section{Limitations of using 45 and Up Study data}

There are some limitations to these data for use by LHDs and PHNs. Although the low response rate (21\%) limits the use of the data for prevalence studies, research has shown that internal comparisons are valid. ${ }^{8}$ Currently, clinical results - such as the results of diagnostic testing - are not available. Establishing diagnoses relies on self-reporting through information provided at baseline or at a follow-up questionnaire, or from the type of care provided - for example, codes for diagnostic testing or reason for hospitalisation. This situation will change as experience in using these data and access to new administrative data collections improve. For example, work is already under way to extract the results of clinical testing from pathology companies. ${ }^{9}$

The preliminary work reported here had the capacity to investigate 12 months of follow-up, because the administrative data were only held to 2009. The facility to include ongoing linkage will enable follow-up of data for at least 10 years. Finally, the capacity to evaluate health service interventions will be limited if exposure measures cannot be identified in the data.

\section{Conclusion}

This paper considered the usefulness of a subset of the 45 and Up Study to explore issues of specific local interest. The 45 and Up Study is a powerful tool that is widely used for health service research. The capacity to link to a wide number of administrative data collections has enabled the creation of comprehensive data collections across national and state jurisdictions.

There are opportunities to use these data to inform health services policy and planning within LHDs and PHNs. Locally, a subset of the 45 and Up Study data will provide a population-based cohort of regional residents that would be expensive to establish. These data have the capacity to provide information on the local community, use of health services (particularly relating to the transfer of care between primary and secondary services) and changes in use over time. These data are relevant to addressing efforts to improve integration of health services and access to preventive care.

The ideas presented in this paper were based on preliminary work undertaken by the Centre for Primary Health Care and Equity on behalf of Sydney LHD, South Eastern Sydney LHD, and Central and Eastern Sydney $\mathrm{PHN}$. Within this region, more than 31000 residents are participating in the 45 and Up Study. Preliminary analysis of the linked data has provided information that is informing discussion about health service policy and practice. The next step in this work is to establish a 45 and Up Primary and Community Health Cohort that will be used to inform services within the participating organisations.

\section{Acknowledgements}

This research was completed using data collected through the 45 and Up Study (www.saxinstitute.org. au/our-work/45-up-study/). The 45 and Up Study is managed by the Sax Institute in collaboration with major partner Cancer Council NSW; and partners the National Heart Foundation of Australia (NSW Division); the NSW Ministry of Health; NSW Government Family \& Community Services - Carers, Ageing and Disability Inclusion; and the Australian Red Cross Blood Service. We thank the many thousands of people participating in the 45 and Up Study. The authors would also like to thank the Australian Government Department of Human Services for access to MBS and PBS data, the NSW Ministry of Health for access to the Admitted Patient Data Collection, the NSW Register of Births, Deaths and Marriages for access to death data, and $\mathrm{CHeReL}$ for facilitating the record linkage. This preliminary work was funded by Sydney LHD and South Eastern Sydney LHD. The conduct of the 45 and Up Study was approved by the University of NSW Human Research Ethics Committee (HREC), and the specific study was covered by the NSW Population and Health Service HREC (10/CIPHS/27). 


\section{Competing interests}

$\mathrm{MH}$ is a board member of the Central and Eastern Sydney PHN.

\section{Author contributions}

The paper has been discussed with members of the project management group, and they are acknowledged. The authors all contributed to the conceptualisation of the paper. EC, EH and $\mathrm{MH}$ developed the outline for the paper; all of the authors commented on the development of the paper; and EC wrote the paper.

\section{References}

1. 45 and Up Study Collaborators, Banks E, Redman S, Jorm L, Armstrong B, Bauman A, et al. Cohort profile: the 45 and up study. Int J Epidemiol. 2008; 37(5):941-7.

2. Australian Government Department of Human Services. Medicare. Canberra: Department of Human Services; 2016 [cited 2016 Apr 28]. Available from: www. humanservices.gov.au/customer/dhs/medicare

3. New South Wales Ministry of Health. Local Health Districts and speciality networks. Sydney: NSW Government; [cited 2016 Apr 28]. Available from: www. health.nsw.gov.au/lhd/pages/default.aspx

4. Australian Government Department of Health. Primary Health Networks. Canberra: Commonwealth of Australia; [cited 2016 Apr 28]. Available from: www.health.gov.au/ internet/main/publishing.nsf/Content/PHN-Home
5. Sydney Local Health District. Sydney Local Health District strategic plan 2012-2017 (revised 2014). Sydney: NSW Government; 2014 [cited 2016 Feb 29] Available from: www.slhd.nsw.gov.au/pdfs/sp12-17.pdf

6. Sydney Local Health District. Aged health, chronic care, rehabilitation and general medicine (ACC\&R) position paper 2013-2018. Sydney: NSW Department of Health; 2013 [cited 2016 Feb 29]. Available from: www.slhd.nsw. gov.au/planning/pdf/ACCR_Clinical_Stream_Position_ Paper.pdf

7. South Eastern Sydney Local Health District. Aged Care Services Plan 2015-2018. Sydney: South Eastern Sydney LHD; 2015 [cited 2016 Feb 29]. Available from www.seslhd.health.nsw.gov.au/Planning_ and_Population_Health/documents/HealthPlans/ FinalAgedCarePlan_22\%20April_2015.pdf

8. Mealing NM, Banks E, Jorm LR, Steel DG, Clements MS, Rogers KD. Investigation of relative risk estimates from studies of the same population with contrasting response rates and designs. BMC Med Res Methodol. 2010;10:26.

9. Jardine M, Foote C, Comino E, Liu B. The impact of chronic disease progression: augmenting 45 and Up with community pathology data. Presented at the Annual 45 and Up Study collaborators' meeting 2015; 2015 Nov 24; Sydney.

\section{Copyright: (c) (i) (2)}

(C) 2016 Comino et al. This article is licensed under the Creative Commons Attribution-NonCommercial-ShareAlike 4.0 International Licence, which allows others to redistribute, adapt and share this work non-commercially provided they attribute the work and any adapted version of it is distributed under the same Creative Commons licence terms. See: www.creativecommons.org/licenses/by-nc-sa/4.0/ 